\title{
Mineraçãåo
}

\section{Utilização de critérios geoestatísticos para comparação de malha de sondagem visando à maximização da quantidade de recursos}

\section{Geostatistical approach for drilling pattern selection based on mineral resource risk assessment}

\section{Diniz Tamantini Ribeiro \\ VALE S.A. \\ Gerência de Modelagem Geológica de Ferrosos diniz.ribeiro@vale.com}

Cid Gonçalves Monteiro Filho

VALE S.A.

Gerência de Modelagem Geológica de Ferrosos cid.monteiro@vale.com

\section{Luis Eduardo de Souza}

Universidade Federal do Pampa

Curso Superior de Tecnologia em Mineração

luissouza@unipampa.edu.br

João Felipe C. L. Costa

Universidade Federal do Rio Grande do Sul

Departamento de Engenharia de Minas

jfelipe@ufrgs.br

Delia Del Pilar Montecinos de Almeida Universidade Federal do Pampa

Curso Superior de Tecnologia em Mineração deliaalmeida@unipampa.edu.br

\section{Resumo}

A proposta básica desse estudo é definir malhas ótimas de sondagem a serem aplicadas em pesquisas futuras de jazidas de minério de ferro. Realizou-se um estudo geoestatístico utilizando-se como parâmetro de otimização, a distribuição das classes dos recursos, obtidas a partir do Índice de Risco (IR), adotado como padrão, na avaliação de depósitos e minas de ferro pela Vale. Esse índice baseia-se no emprego da krigagem da indicatriz de minério para classificação dos recursos, avaliando-se, então, a influência da variação da malha de sondagem na categorização dos mesmos, pela criação de malhas regulares de sondagem "virtuais" com espaçamentos diversos. Estudos comparativos foram realizados em minas existentes e em projetos, tanto no Quadrilátero Ferrífero, quanto em Carajás, obtendo-se malhas de sondagem indicativas por província, sendo que, para novas áreas, criou-se uma metodologia de pesquisa sequencial, com adensamento gradativo da malha de sondagem e avaliação dos riscos em cada etapa.

Palavras-chave: Malha de sondagem, geoestatística, otimização amostral.

\begin{abstract}
Mineral deposit assessment is based on estimates of the attributes within the ore body, where the sampling of these attributes is usually obtained through cores from diamond drill holes. Due to the high costs associated with drilling and sampling, the amount of drill holes is limited and their locations must be correctly defined to simultaneously maximize the uncertainty reduction related to the estimates and the confidence of the resource and reserve inventories. Having posed the problem, this article presents a methodology for defining the ideal drill-hole grids for optimizing the distribution of resources according to their measured, indicated, and inferred classes. The method combines the estimation error and geological continuity through an indicator kriging approach. This methodology is currently used as a benchmark in all the ferrous resource classification done at the Vale Ferrous mine planning department, and a comprehensive case study was conducted in two different iron ore deposits demonstrating its robustness and applicability in any mineral inventory assessment.
\end{abstract}

Keywords: Sampling location, goestatistics, drilling optimization. 


\section{Introdução}

Durante os estágios iniciais de desenvolvimento de uma nova mina, um depósito pode ser considerado como conhecido do ponto de vista da exploração, se a informação disponível for suficiente para dar suporte ao planejamento de longo prazo, sem nenhum desvio significativo em relação ao depósito real. Nesse sentido, dois problemas podem ocorrer: (i) qualidade do minério (teores) superestimada ou subestimada e (ii) erro de volume local na interpretação geológica (estéril ser indicado como minério ou vice-versa).

Enquanto o primeiro problema depende, basicamente, do espaçamento utilizado na amostragem e da garantia de qualidade das amostras, podendo ser adequadamente tratado com a utilização

\section{Metodologia}

\section{Krigagem de indicadores}

De acordo com Rivoirard (1994), a expressão de uma Função Aleatória pode ser simplificada pela utilização de indicadores. Os indicadores são, frequentemente, utilizados, quando um dado teor de corte é empregado (Journel, 1983). As amostras cujo teor estiver acima do teor de corte assumem o valor um (1) e aquelas com teor abaixo do teor de corte assumem o valor zero (0).

A complexidade geológica pode ser representada pelo variograma dos indicadores. A Figura 1 apresenta dois modelos variográficos empíricos escolhidos para demonstrar o efeito das geometrias dos corpos de minério. O corpo A é mais contínuo e seu variograma de indicadores normalizado, com patamar unitário, de técnicas geoestatísticas tradicionais, o segundo problema depende, tanto do espaçamento das amostras, quanto da complexidade geológica do corpo de minério.

A proposta básica desse estudo é apresentar uma solução para ambos os problemas através da identificação de áreas com alto risco na interpretação geológica, levando-se em conta, tanto a continuidade geológica, ligada ao conceito de homogeneidade do domínio de interesse (Sinclair \& Blackwell, 2002), quanto a densidade de amostragem, ligada à regionalização do minério.

Com esse critério de avaliação de risco, é possível definir malhas ótimas de sondagem a serem aplicadas em pesquisas futuras de novas jazidas e/ou minas de ferro existentes. Em geral, a identifica- ção das áreas de risco é obtida de maneira empírica, baseando-se apenas na experiência do geólogo. No entanto, devido à subjetividade, essa definição nem sempre é considerada durante a elaboração de um plano de lavra, podendo, então, levar a resultados inesperados. Nesse sentido, um procedimento prático e robusto deveria, obrigatoriamente, atender as seguintes premissas:

i. Evitar diferentes interpretações para o mesmo problema e permitir sua incorporação nos planos de lavra.

ii. Facilitar a compreensão e a implementação.

iii. Levar em consideração as complexidades geológicas dos corpos de minério e as características intrínsecas de cada depósito. apresenta um alcance maior do que o correspondente variograma do corpo $\mathrm{B}$.

É importante perceber que, ao se adotar um variograma com patamar unitário, elimina-se o efeito de variação do patamar sobre a variância de krigagem do bloco e, com isso, adota-se um padrão único de "erro de estimativa máximo". A variância de krigagem é função do modelo variográfico, da geometria do bloco e da distribuição espacial das amostras. Fixando-se o tamanho do bloco e o patamar, a variância de krigagem terá forte correlação com a densidade de amostragem.

A krigagem ordinária de indicadores leva em conta o suporte dos blocos e o resultado para cada bloco será uma média ponderada dos indicadores das amos- tras. O referido resultado, por definição, assume apenas como valor mínimo 0 (zero) e como valor máximo 1 (um), exceto para alguns poucos blocos com pesos de krigagem negativos.

Os blocos com resultado da krigagem igual a um têm alta probabilidade de serem compostos de "minério", enquanto blocos que apresentam valores zero têm alta probabilidade de serem compostos por outros materiais. Os valores intermediários entre zero e um indicam a probabilidade do dado bloco ser minério. Se os resultados da krigagem indicam a litologia mais provável, a variância de krigagem indica o grau de confiança dessa hipótese e, assim, ambos os parâmetros são considerados na metodologia proposta.

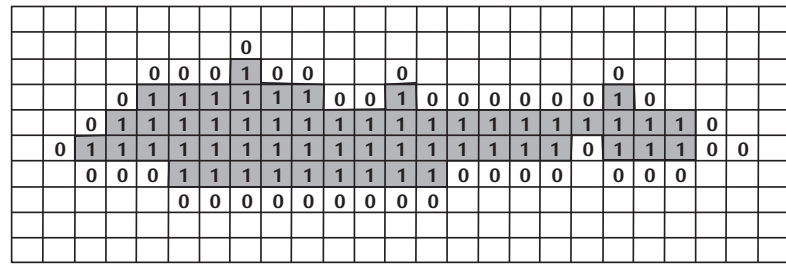

Corpo A

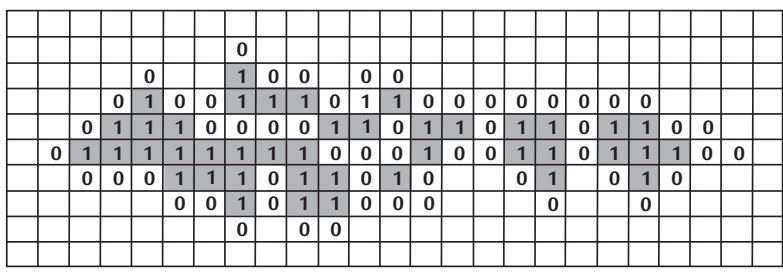

Corpo B

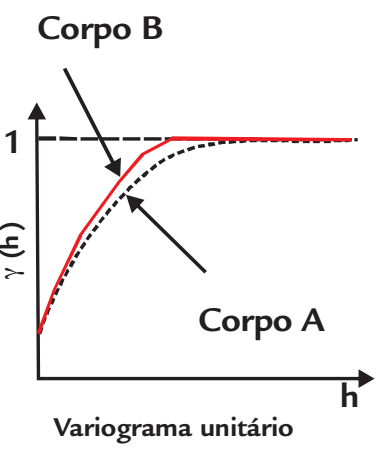

Figura 1

Exemplo esquemático de variogramas

de indicadores para o corpo $A$

(em preto) e para o corpo B

(em vermelho). 


\section{O índice de risco (IR) e as classes de recursos}

$\mathrm{O}$ índice de risco, apresentado por Amorim e Ribeiro (1996), surgiu como ferramenta alternativa para a classificação de recursos minerais, com a proposta de combinar a incerteza geológica e a variância de krigagem. Diversos outros métodos de classificação de recursos estão

Para cada bloco, é possível avaliar quatro situações, conforme ilustrado na Figura 2A. Os blocos cujo par [(1 - $\left.\mathrm{I}_{\mathrm{k}}\right)$, $\left.\mathrm{S}_{\mathrm{ik}}^{2}\right]$ ficar localizado na região I mostram alta continuidade geológica e baixa variância de krigagem, indicando uma área com baixo risco; a região II mostra alta continuidade geológica e alta variância, indicando um risco devido à ausência de

Figura 2

Exemplo da metodologia de classificação, $\operatorname{com}(A)$ as classes de recursos e (B) o vetor do índice de risco (IR).

Utilização do índice de risco como critério de classificação de recursos.

\section{Otimização de malha de sondagem}

Os benefícios associados à amostragem estão relacionados, não somente com seu número adicional, mas, claramente, com a configuração espacial adotada. Em Koppe et al. (2009), por exemplo, é estudada a relação entre os esquemas de locação de amostras com a redução da incerteza sobre uma função de transferência.

Na metodologia adotada, nesse trabalho, a partir da definição do critério de classificação, a distribuição dos recursos por categoria passa a se constituir um parâmetro a ser utilizado na definição discutidos em trabalhos como Mwasinga (2001) e Souza et al. (2009).

De maneira simplificada, o IR pode ser calculado a partir de dois parâmetros: $\left[1-\mathrm{I}_{\mathrm{k}}\right]$ e $\left[\mathrm{S}_{\mathrm{ik}}^{2}\right]$, onde $\mathrm{I}_{\mathrm{k}}$ é a krigagem do indicador "minério" para o suporte u e $S_{\mathrm{ik}}^{2}$ é a respectiva variância

$$
R I(u)=\sqrt{\left[1-I_{k}^{*}(u)\right]^{2}+\left[S_{i k}^{2}(u)\right]^{2}}
$$

informação; a região III apresenta baixa continuidade geológica e baixa variância, indicando risco devido à proximidade de contato minério/estéril; a região IV apresenta uma baixa continuidade geológica e baixa variância, indicando alto risco devido a ambas as razões. A seção horizontal apresentada na Figura 2 exemplifica os resultados finais.

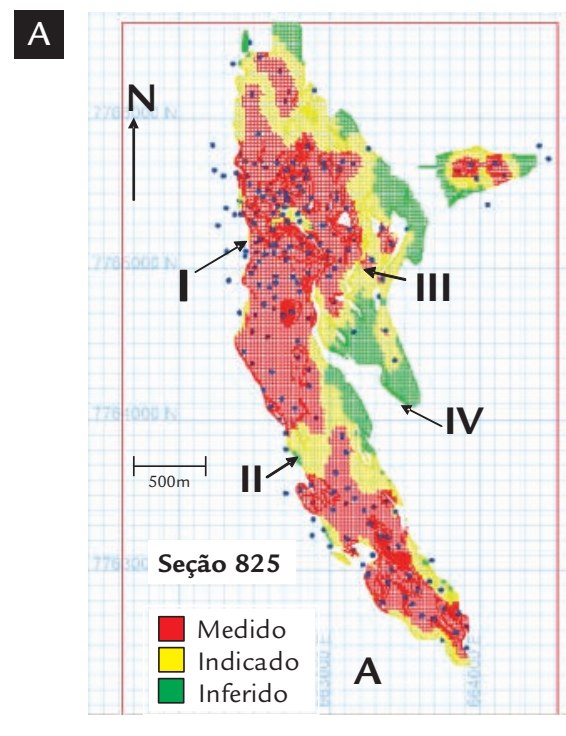

B

\begin{tabular}{c|c}
\hline Categoria & Índice de Risco \\
\hline Medido & $\mathrm{RI}<0.6$ \\
\hline Indicado & $0.6<\mathrm{RI}<0.9$ \\
\hline Inferido & $0.9<\mathrm{RI}$ \\
\hline
\end{tabular}

de krigagem (usando variograma com patamar unitário).

No plano cartesiano, formado pelo par $\left[1-\mathrm{I}_{\mathrm{k}}\right]$ e $\left[\mathrm{S}_{\mathrm{ik}}^{2}\right]$ (Figura $2 \mathrm{~b}$ ), o vetor IR é a "distância" até a origem e, portanto, o valor pode ser obtido através da seguinte expressão:

Calculado o valor do índice de risco para cada bloco, o mesmo pode ser utilizado como critério de classificação, atribuindo-se níveis para cada classe de recurso (Tabela 1). Nesse ponto, é importante que se faça uma análise detalhada com objetivo de se obter uma coerência com a expectativa geológica.

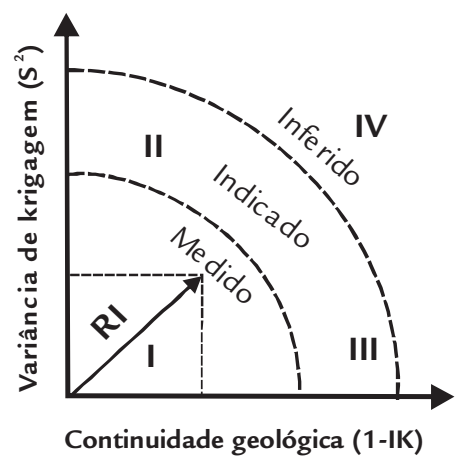

da malha a ser praticada. Assim, na metodologia proposta, são criadas malhas regulares de sondagem "virtuais" com espaçamentos diversos. A partir dessas malhas, novas krigagens com adição desses pontos de observação "virtuais" da variável indicatriz de "minério", para classificação dos recursos de diversos depósitos ou minas, são efetuadas. Avaliase, então, a influência da variação da malha de sondagem na categorização dos recursos geológicos.

De maneira sucinta, a seguir estão listadas as principais etapas desenvolvi- das durante a execução do estudo.

i. Seleção de minas ou depósitos representativos dos tipos de minério que ocorrem no Quadrilátero Ferrífero e na Província de Carajás.

ii. Criação de malhas de sondagem regulares cortando todo o corpo de minério interpretado e zona de borda não mineralizada ou de baixo teor.

iii. Classificação das amostras virtuais em indicatrizes de minério de acordo com a interpretação geológica do modelo (na zona de minério a indicatriz é igual a $1 \mathrm{e}$, na zona de estéril ou mi- 
neralizada de baixo teor, a indicatriz igual a 0).

iv. Krigagem da indicatriz usando as amostras "virtuais" obtidas no modelo geológico interpretado com o variograma utilizado na classificação do recurso: tipos associados à formação ferrífera, exceto itabiritos compactos, no Quadrilátero Ferrífero, e tipos hematíticos, na Província de Carajás.

v. Cálculo das proporções de classes de recursos medido, indicado e inferido, de acordo com o método de classificação, para cada malha virtual.

vi. Construção de gráficos comparando malhas de sondagem e proporção de classes de recursos.

A Figura 3 ilustra um exemplo de aplicação da metodologia em um depósito de ferro localizado no Quadrilátero Ferrífero. Com uma malha de sondagem
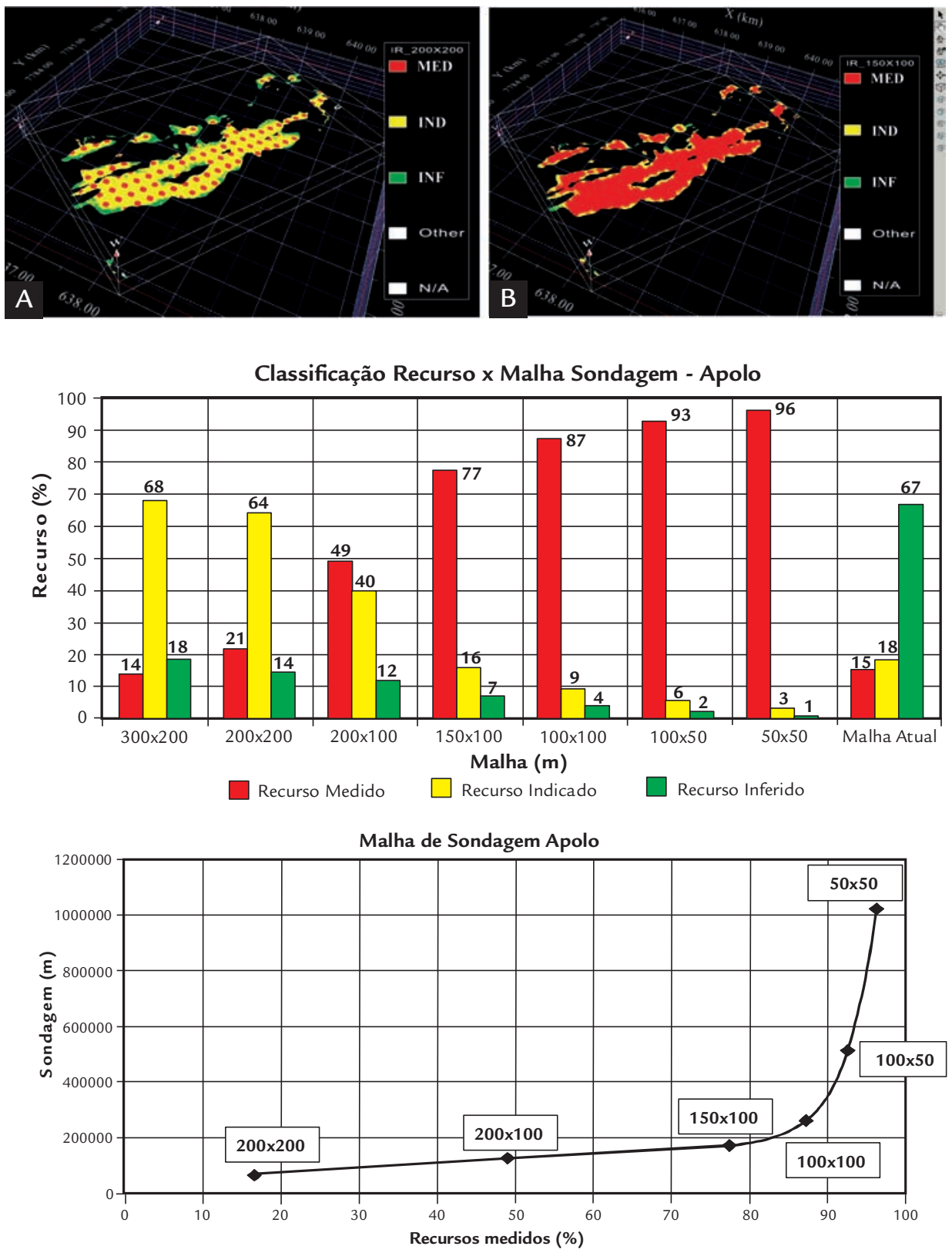

Na Figura 6, são apresentados os recursos seriam classificados como medidos e $65 \%$ seriam recursos indicados. Praticando-se uma malha de $150 \times 100$ m (Figura 3B), 77\% dos recursos seriam classificados como medidos e $16 \%$ como indicados.

A aplicação de diversas malhas de sondagem pode ser testada, obtendo-se, simultaneamente, uma diferente distribuição de recursos por categoria, conforme ilustrado na Figura 4. Dessa forma, a tomada de decisão se baseia na adoção da malha capaz de fornecer a distribuição alvo da empresa para um dado depósito ou mina.

Da mesma forma, é possível obter uma estimativa da quantidade de recursos a serem classificados como medidos em função da quantidade de metros perfurados (Figura 5).

Figura 3

Distribuição espacial, por categoria de recurso, com a aplicação das malhas

(A) $200 \times 200 \mathrm{~m}$

(B) $150 \times 100 \mathrm{~m}$.

Figura 4

Análise comparativa das distribuições de recursos por categorias para diversas malhas de sondagem testadas para o depósito do Quadrilátero Ferrífero.

Figura 5

Distribuição dos recursos medidos em função dos metros perfurados. 
Figura 6 Análise comparativa das malhas na classificação de recursos para a jazida de Conta História.

\section{Conclusões}

O emprego sistemático da metodologia apresentada para minérios de ferro tem mostrado que as malhas definidas como adequadas são, em geral, mais abertas ou coincidentes com aquelas praticadas nas pesquisas atuais.

Um cuidado especial, na aplicação dessa metodologia, deve ser adotado
Estudo de Malhas Conta História (cth_1208.bmf)

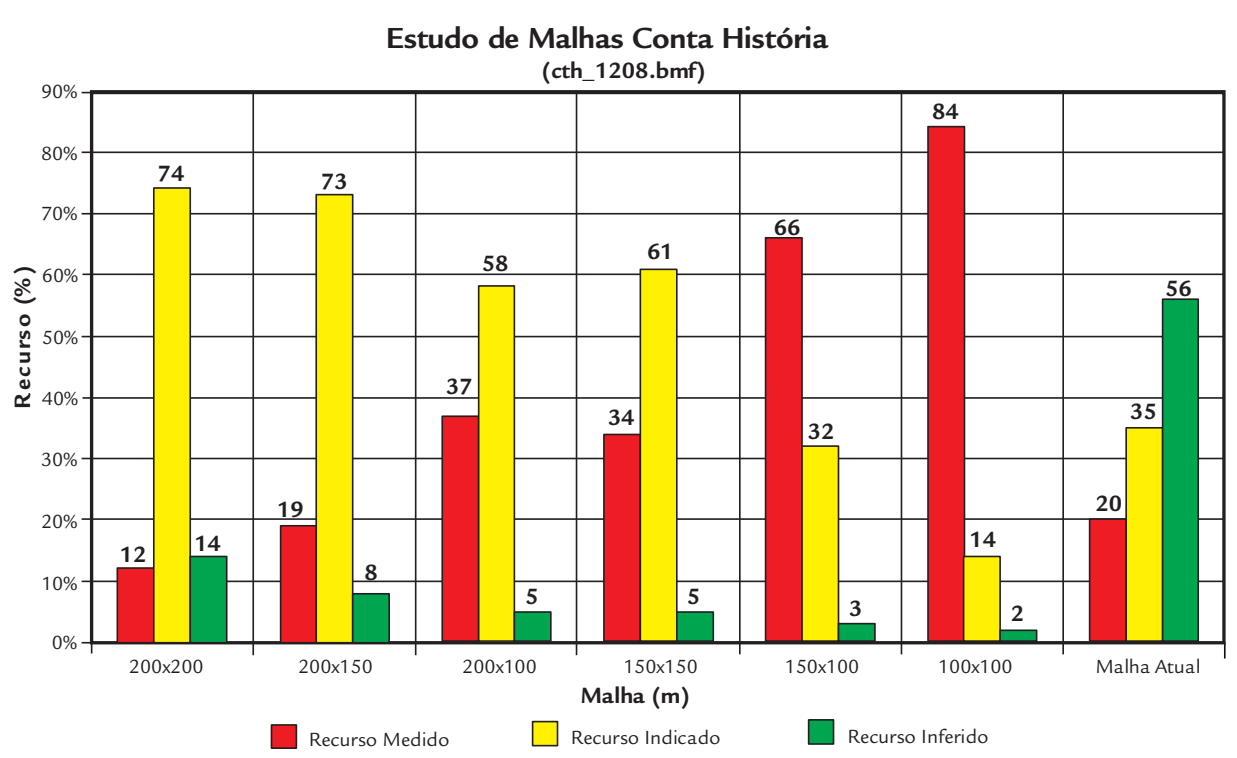

em novos depósitos. Nesses depósitos a pesquisa deve ser dividida em etapas, diluindo-se os investimentos no tempo e evitando-se sondagens excessivas em áreas de pouco interesse. A proposta de sequenciamento de sondagem está resumida na Figura 7.

É importante salientar, no en- tanto, que a opção pela divisão da pesquisa mineral em etapas só será válida se estiver de acordo com os objetivos estratégicos da empresa, já que questões de atraso no licenciamento ambiental, por exemplo, podem inverter a ordem de prioridade proposta nesse estudo.
Figura 7

Fluxograma sugerido para pesquisas em depósitos novos.

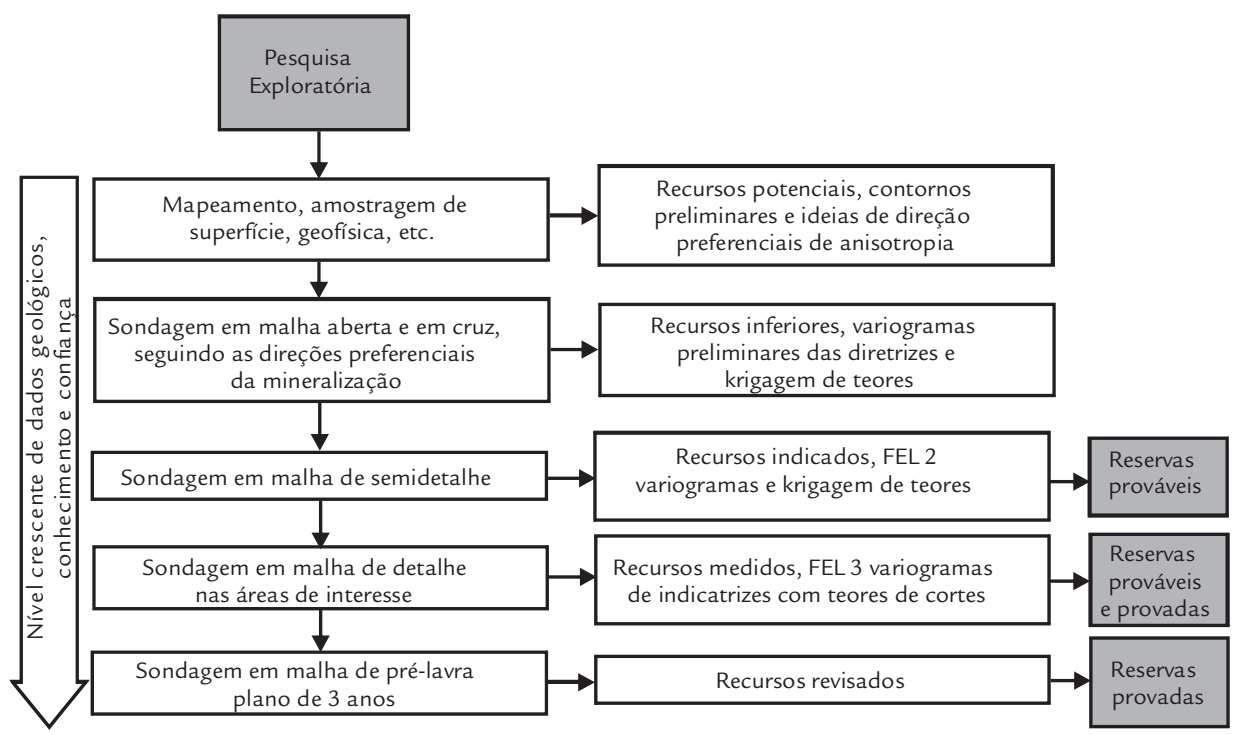

\section{Referências bibliográficas}

AMORIM, L.Q., RIBEIRO, D. T. An Useful Ore Reserve Classification Criterion Based on Indicator Kriging, In: Proceedings of Mine Planning and Equipment Selection. In: HENNIES, A. da Silva, CHAVES. (Ed.). Balkema, 1996. p. 117-121.

JOURNEL, A.G. Non-parametric estimation of spatial distributions. Mathematical Geology, v. 15, n. 3, 1983, p. 445-468.

KOPPE, V.C., COSTA, J. F. C. L., KOPPE, J.C. Algorithm to help additional drilling location. Proceedings, APCOM 2009, The Canadian Institute of Mining, Metallurgy and Petroleum, Vancouver, 2009, p. 87-93.

MWASINGA, P.P. Approaching resource classification: general practices and the integration. In: INTERNATIONAL SYMPOSIUM ON COMPUTER 
APPLICATIONS IN THE MINERAL INDUSTRIES (APCOM'2001), 29.

Proceedings... Beijing, China, 2001, p. 97-104.

RIVOIRARD, J. Introduction to disjunctive kriging and non-linear geostatistics. Oxford: Clarendon Press, 1994.

SINCLAIR, A.J., BLACKWELL, G.H. Applied mineral inventory estimation. Cambridge: University Press, 2002.

SOUZA, L.E., COSTA, J.F.C.L., KOPPE, J.C. A geostatistical contribution to assess the risk embedded in resource classification methods. In: IRON ORE CONFERENCE 2009, Proceedings... Perth, Australia, 2009.

Artigo recebido em 18 de maio de 2010. Aprovado em 30 de setembro de 2011. 